\title{
Effectiveness of Antenatal Exercise Intervention Using Behavioral Theory on Exercise Adherence Among Antenatal Women: Research Protocol
}

\author{
Nur Farhana Md Yunus ${ }^{1,2 *}$ Rosliza Abdul Manaf ${ }^{1}$ Hayati Abd Kadir ${ }^{1}$ Suriani Ismail ${ }^{1}$ \\ Parwathi Alagirisamy ${ }^{3}$ \\ 1 Department of Community Health, Faculty of Medicine and Health Sciences, Universiti Putra Malaysia, 43400 \\ Serdang, Selangor, Malaysia \\ 2 Centre for Physiotherapy Studies, Faculty of Health Sciences, Universiti Teknologi MARA, Kampus Puncak Alam, \\ 42300 Selangor, Malaysia \\ 3 KPJ Damansara Specialist Hospital, Damansara Utama, 47400 Petaling Jaya, Selangor, Malaysia \\ *Corresponding author. Email: gs58667@student.edu.my
}

\begin{abstract}
Antenatal exercise (ANE) is one of physical activity recommended for antenatal women as the benefits outweigh the risks. Despite knowing the benefits of exercises, most antenatal women are less active during pregnancy. Addressing women's attitude and barriers to exercise are crucial in designing intervention, it should be facilitated by a theory. Hence, this study aims to investigate the effectiveness of antenatal exercise intervention using behavioral theory on exercise adherence among antenatal women. This study will be a cluster randomized controlled trial (CRCT) in six primary health clinics with total sample estimation is 322. A two arm CRCT will be applied, a control group will be received standard antenatal care only while intervention group will be received 12 weeks ANE intervention and standard antenatal care. The ANE intervention module based on behavioral theory is the main study instrument, questionnaires on exercise adherence, perceived benefits, perceived barriers, exercise self-efficacy and HRQOL will be used in this study. Repeated measures logistic regression analysis using generalized estimating equations (GEE) will be used to evaluate the odds ratios of dependent variables between the groups at three-time points. All statistical tests are based on two-tailed test and the level of significance, alpha $(\alpha)$ is set at 0.05 . The expected findings of this study will be a significant difference on dependent variables in intervention than control group at two time point post intervention. It could also improve antenatal women's adherence towards ANE, hence promote active lifestyles and reduce maternal implications.
\end{abstract}

Keywords: antenatal exercise, behavioral theory, exercise adherence, self-efficacy, antenatal women.

\section{INTRODUCTION}

Pregnancy poses unique challenges for women. This is a period when physiological, emotional and social changes occur. It is essential during this period to implement lifestyle changes that will benefit maternal and foetal well-being. This opportunity is identified as a 'teachable moment' in a woman's life, in which women are responsive to change behaviours that can benefit their health and baby's health [1]. Physical activity is recommended for antenatal mothers as the benefits outweigh the risks. The ACOG updated in 2015 recommended that healthy antenatal women exercise at moderate-intensity aerobic activity at least 150 minutes per week during pregnancy and the postpartum period [2].

Antenatal exercise, also called as prenatal or maternal exercise, is any kind of exercise that is considered safe during pregnancy. These specific exercises include aerobics, pelvic floor, stretching, muscle strengthening, abdominal and back care exercise, cycling, swimming, relaxation and breathing exercise [3-4]. Other types of exercises found to be safe and beneficial during pregnancy are walking, jogging, yoga and pilates for those uncomplicated pregnant women. 
It helps to reduce the need for obstetric intervention or caesarean section during labour and delivery [5]. However, pregnant women are likely to be physically inactive due to their need to adjust to substantial physiological and psychological changes during pregnancy [6], thus present with a discomforts which might lead to the barrier in exercise.

Despite knowing the benefits of antenatal exercises, previous studies have shown that most antenatal women are less active during pregnancy [4, 7]. Globally, $60 \%$ of US women not engaged in exercise during pregnancy [8], $52.4 \%$ in the United Kingdom (UK), $78.5 \%$ in Ireland, $88.9 \%$ in China [8-9] and $16.4 \%$ in Saudi [10]. Likewise, in Malaysia has the highest prevalence of low physical activity rates (58\%) among females [11]. A recent study reported $64.5 \%$ physical inactivity among antenatal women [12]. In the long term, if these figures persist, it will result in increasing number of chronic diseases as well as increasing burden to the government in managing the non-communicable diseases (NCD) such as obesity, diabetes and hypertension.

Lack of physical activity has been identified as fourth leading causes for global mortality [13]. Indeed, it is a particular concern for antenatal women as they prone to have pregnancy related conditions, thus regular physical activity has been recommended by the ACOG to encourage antenatal women to be active [14]. Hence, encourage antenatal exercise during pregnancy is one of the approach that could be implemented to improve maternal health.

Essentially, attitude and practice of exercise, barriers and predictors to practice need to determine before developing appropriate intervention to encourage antenatal women to engage in exercise. Addressing women's attitude and barriers to exercise are crucial in designing antenatal exercise strategies, it should be facilitated by a theory or model.

One of the issues related to exercise is the compliance or adherence to such activity that may be influenced or determined by several factors. Adherence is an important aspect in practicing exercise. A number of factors influence exercise adherence during pregnancy such as tiredness, lack of feeling to exercise, lack of time, lack of knowledge and support and pregnancy discomforts were the most implicated factors to practice ANE [15-16].

Lack of study based on the information-motivationbehavioral (IMB) model in the development of physical activity intervention during pregnancy particularly in adherence. To improve participation in antenatal exercise, intervention need to be developed through theory-based approaches to facilitate behaviour change in exercise adherence and improve HRQOL.
Hence, the general objective of this study is to investigate the effectiveness of ANE intervention using IMB model on exercise adherence among antenatal women. Meanwhile the specific objectives are: (1) to determine the level of knowledge, attitude, practice and barriers of antenatal exercise among antenatal women in primary healthcare, (2) to identify the predictors to practice exercise among antenatal women in primary healthcare, (3) to develop and validate ANE intervention module, (4) to determine the effectiveness of ANE intervention by measure the differences in exercise adherence, perceived benefits, perceived barriers and exercise self-efficacy and HRQOL at baseline, immediately post intervention and 1 month post intervention within and between the intervention and control group, controlling the covariates.

\section{MATERIAL \& METHOD}

This study will comprise of three phases with the aim to obtain the general and specific objectives accordingly. Phase 1 is a cross sectional study will be conducted in seven (7) government primary health clinics under the administration of the Kuala Selangor Health District. Total sample estimation will be 614. Proportionate stratified random sampling will be applied in this study to execute sampling. The adapted KAP questionnaire will be used in this phase.

Phase 2 will be develop ANE intervention module based on findings in phase 1 integrated with IMB model constructs. The ANE intervention content will be validated beforehand to avoid presenting unclear medical terms and to simplify the language as needed.

Phase 3 will be a cluster randomized controlled trial (CRCT) with single blind, will be conducted in six (6) primary health clinics will be selected based on higher number of antenatal women check-up visit in Kuala Selangor Health District. Total sample estimation will be 322. A two arm CRCT will be applied, a control group will received standard antenatal care only while intervention group will received 12 weeks antenatal exercise intervention and standard antenatal care. The ANE intervention module is the main instrument, questionnaires assessing the exercise adherence, perceived benefits, perceived barriers, exercise selfefficacy and HRQOL will be used in this study.

\section{DATA COLLECTION}

In considering the pandemic Covid-19 issue, the researcher will take into account the data collection will be collected via online for phase 1 . There will be an informative advertisement about this research with a registration link or QR code to join this research through flyers and banner at the respective clinics. The participants will sign e-consent to participate in this 
study. After they agreed to participate, they will have to answer screening questions for the inclusion and exclusion criteria. Those who are eligible will be contacted by the researcher and receive a link to answer the questionnaire through google form.

In phase 2, the ANE intervention modules will be developed in the Malay language, will be validated beforehand to avoid presenting unclear medical terms and to simplify the language as needed. The applicable strategies such as short lecture video, interactive ANE video, short messaging and multimedia messaging service (MMS) technology to deliver the intervention content, guided intervention session to enable participants to obtain information, motivation and behavioral skills to the respondents. The follow up intervention strategies will be given according to the session as a progression and feedback by phone call or via google meet. The participant will get the feedback and suggestions for improvement once a month following their antenatal care appointment.

In phase 3 , the eligible participants who consented to be involved in this study will be informed of the study protocol, and written consent will be obtained after they understood the information in the protocol. The process of explaining the study protocol and obtaining written consent from the participants will be conducted by the researcher via online.

The participants in the intervention group will be received 12 weeks antenatal intervention module and standard antenatal care while the control group will be received standard antenatal care only. The intervention group will be received ANE module in six sessions (biweekly) during the three-month intervention via online approaches. The control group will be received standard antenatal care by the HCPs in the respective clinics.

\section{STATISTICAL TEST/DATA ANALYSIS}

The descriptive statistics to describe sociodemographic and socioeconomic characteristics of the respondents are mean, standard deviation, interquartile range and percentage will be used. While the bivariate analysis will be used a Chi-square test to measure the associations between independent and dependent variables. Subsequently, binary logistic regression analysis will be conducted to measure predictors of KAP among antenatal women. Normality test will be tested before the inferential statistical test will be run.

Repeated measures logistic regression analysis using generalized estimating equations (GEE) will be used to evaluate the odds ratios of adherence, perceived benefits, perceived barriers and exercise self-efficacy and HRQOL between the intervention groups and control groups at three-time points. The level of significance at 0.05 and 95\% confidence interval (CI) will be used to get a significant result. All statistical tests are based on twotailed test and the level of significance, alpha $(\alpha)$ is set at 0.05 .

\section{EXPECTED RESULT}

There will be a significant differences in intervention group than control group on subject's adherence, perceived benefits, perceived barriers and exercise selfefficacy and HRQOL at immediately post 12 week intervention and 1 month post intervention. The findings of this study could improve antenatal women's selfefficacy and adherence towards antenatal exercise without relying on the healthcare providers advices. Moreover, HRQOL of the antenatal women could also be improved. Hence, it will promote active lifestyles and reduce maternal implications.

\section{AUTHORS' CONTRIBUTIONS}

N.F.M.Y - conceived of the research idea, will conceived and planned the intervention, will be design and carried out the intervention, wrote the manuscript with support from R.A.M, H.K and S.I.

R.A.M - supervised the research, provided critical feedback and helped shape the research, analysis and manuscript encouraged N.F.M.Y to investigate the outcomes and supervised the findings of this research.

H.K - helped supervise the research, will be verified the analytical methods

S.I - helped supervise the research, provided critical feedback and helped shape the research, analysis and manuscript.

P.A - contributed to the design and implementation of the research

All authors discussed the results and commented on the manuscript.

\section{ACKNOWLEDGMENTS}

The authors wish to thank Universiti Putra Malaysia (UiTM) for administrative support, primary health clinics staff and participants who will be involved in this study.

\section{REFERENCES}

[1] S. Phelan, Pregnancy: a "teachable moment" for weight control and obesity prevention. American Journal of Obstetrics and Gynecology, 2010. DOI: https://doi.org/10.1016/j.ajog.2009.06.008

[2] M.L. Birsner, C. Gyamfi-Bannerman, Committee on Obstetric Practice Physical Activity and Exercise During Pregnancy and the Postpartum Period, ACOG COMMITTEE OPINION Number 804, 2015, pp.178-188. 
[3] ACOG Committee Opinion: Exercise during Pregnancy and the Postpartum Period, 267, 2002. DOI: $10.1016 / \mathrm{s} 0029-7844(01) 01749-5$

[4] C.E. Mbada, O.E Adebayo, A B. Adeyemi, O.O Arije, O.O. Dada, O.A. Akinwande, ....I.A, Alonge, Knowledge and Attitude of Nigerian Pregnant Women towards Antenatal Exercise : A Cross-Sectional Survey. ISRN Obstetrics and Gynecology, 2014.

[5] K. Melzer, Y. Schutz, M. Boulvain, B. Kayser, Physical activity and pregnancy: Cardiovascular adaptations, recommendations and pregnancy outcomes, Sports Medicine, 2010. DOI: https://doi.org/10.2165/11532290-00000000000000

[6] D.S. Downs, L. Chasan-Taber, K.R. Evenson, J. Leiferman, S.Yeo, Physical Activity and Pregnancy: Past and Present Evidence and Future Recommendations, Res $Q$ Exerc Sport, 2012, 83(4), pp.485-502.

[7] K.R. Evenson, K. Borodulin, S.Aytur, Physical Activity Beliefs, Barriers, and Enablers among Postpartum Women, Journal of Women's Health, 2009, 18(12), pp.1925-1934.

[8] K.R. Hesketh, K.R. Evenson, Prevalence of U.S. Pregnant Women Meeting 2015 ACOG Physical Activity Guidelines. American Journal of Preventive Medicine, 2016, 51(3), e87-e89. DOI: https://doi.org/10.1016/j.amepre.2016.05.023.

[9] A. Gaston \& A. Cramp, Exercise during pregnancy: A review of patterns and determinants. Journal of Science and Medicine in Sport, 14(4), 299-305.

https://doi.org/10.1016/j.jsams.2011.02.006

[10] G.M. Youbi \& T.Elsaid, Knowledge, attitude, and practices on exercise among pregnant females attending $\mathrm{Al}$ - Wazarat Health Center, Riyadh , SaudiArabia,2020.DOI:https://doi.org/10.4103/jf mpc.jfmpc.

[11] National Health and Morbidity Survey, Volume II: Non-Communicable Diseases, Risk Factors \& Other Health Problems, 2015.[Online]. Available: https://www.moh.gov.my/moh/resources/nhmsrep ort2015vol2.pdf, [Accessed: Aug. 31, 2020].

[12] N.Y.M.Yusof, N.A.M. Zulkefli, H.S.Minhat, N. Ahmad, Predictors of Physical Inactivity Among Antenatal Women: A Systematic Review, Malaysian Journal of Medicine and Health Sciences, 2020, 16(4), pp. 317-324.

[13] World Health Organisation (WHO), Physical activity in the Western Pacific, 2017. [Online]. Available:https://www.who.int/westernpacific/hea lth-topics/physical-activity, [Accessed: Aug.31, 2021].
[14] R. Artal, M.O'Toole, Guidelines of the American College of Obstetricians and Gynecologists for exercise during pregnancy and the postpartum period, $\mathrm{Br} J$ Sports Med, 2008. DOI: http://dx.doi.org/10.1136/bjsm.37.1.6

[15] C. P. Ribeiro, \& H. Milanez, Knowledge, attitude and practice of women in Campinas, São Paulo , Brazil with respect to physical exercise in pregnancy : a descriptive study, 2011, pp. 1-7.

[16] C.E. Mbada, O.E Adebayo, O. E. Adebayo, T.O Awotidebe, F.A. Faremi, M.O. Oginni, A.O. Ogundele, A.I. Emechete, Practice and Pattern of Antenatal and Postnatal Exercise among Nigerian Women: A Cross-Sectional Study. International Journal of Women's Health and Reproduction Sciences, 2015, 3(2),pp. 93-98. DOI https://doi.org/10.15296/ijwhr.2015.18 